\title{
Chronic idiopathic axonal neuropathy and pain, treated with the endogenous lipid mediator palmitoylethanolamide: a case collection
}

This article was published in the following Dove Press journal:

International Medical Case Reports Journal

12 September 2013

Number of times this article has been viewed

\section{J M Keppel Hesselink}

Faculty of Medicine, University Witten/Herdecke, Witten, Germany

Correspondence: J M Keppel Hesselink Spoorlaan 2a, 3735 MV Bosch en Duin, the Netherlands

Email jan@neuropathie.nu

\begin{abstract}
Chronic idiopathic axonal polyneuropathy is a frequent diagnosis in patients suffering from idiopathic polyneuropathy and neuropathic pain. No guidelines exist on how to treat these patients. To date, there are no results available from randomized clinical trials, and mostly classical neuropathic analgesics are prescribed, such as amitriptyline and gabapentine. However, the usefulness of these drugs is limited, as many patients remain in pain despite treatment, or suffer debilitating side effects. Palmitoylethanolamide (PEA) is a new analgesic compound, tested in more than 4,000 patients in various clinical trials in a variety of patients suffering from various neuropathic pain states. It is available in Europe and the USA as a food supplement under the brand name PeaPure, and it is available for medical purposes in Italy and Spain under brand names Normast and Pelvilen. We present a case series of seven patients with an electrophysiological confirmed diagnosis of chronic idiopathic axonal polyneuropathy, suffering from neuropathic pains, mostly refractory to previous analgesics. In all these patients, PEA reduced pain significantly, without side effects. PEA can be administered in addition to other analgesics, without negative drug-drug interactions, or can be used as a stand-alone analgesic. Due to a favorable ratio between efficacy and safety, PEA should be considered more often as a treatment for neuropathic pain.
\end{abstract}

Keywords: CIAP, polyneuropathy, treatment, neuropathic

\section{Introduction: idiopathic axonal neuropathy}

In the last century, various names have been suggested for axonal polyneuropathy without a clear cause, such as "late-life chronic peripheral neuropathy of obscure nature" in $1982^{1}$ and "chronic polyneuropathy of undetermined cause" in 1984 . $^{2}$ Notermans et $\mathrm{al}^{3}$ coined the term chronic idiopathic axonal polyneuropathy (CIAP), which has been adopted worldwide. CIAP should be differentiated from CAIP, chronic axonal inflammatory polyneuropathy. ${ }^{4}$ Vrancken et $\mathrm{al}^{5}$ complained in 2004 that even though CIAP has been clearly described and delineated, no adequate randomized or quasirandomized controlled clinical treatment trials had been performed. In their absence, there was no proven efficacious drug therapy. This still seems to be the case. CIAP is not yet recognized by pharmaceutical companies as a target indication, most probably due to the absence of insight into its pathogenesis and the pathophysiological mechanisms leading to CIAP. Therefore, causal treatment options are absent; to date, only symptomatic treatment seems possible. There are, however, many patients suffering from this disorder of the peripheral nervous system; an estimated 5-8 million Americans, comprising about one-third of all patients with neuropathy. ${ }^{6}$ Although the pathogenesis and etiology of CIAP remains a mystery, it was recently suggested by 
Visser et $\mathrm{al}^{7}$ that metabolic syndrome could be a risk factor related to its pathogenesis.

In our clinic, we regularly find a deficient vitamin D3 status in CIAP patients suffering from chronic neuropathic pain. Furthermore, a number of patients diagnosed with CIAP have used statins for a long period of time, and CIAP symptoms emerged after this therapy. There is, however, no consensus yet as to whether statins might cause CIAP or whether statins might contribute to its pathogenesis. ${ }^{8}$ Statins have, however, been described as neurotoxic. ${ }^{9,10}$ For the time being, the etiology and the pathogenesis of CIAP remains an enigma, and as a "waste basket" diagnosis, we need to understand patients diagnosed with CIAP might suffer from different diseases.

Pain is a symptom frequently seen in CIAP patients; perhaps more than half of all patients complain about pain, a pain mostly described as nagging and annoying. ${ }^{11}$ Small fiber neuropathy and neuropathic pain also seems to occur in CIAP patients. ${ }^{12}$

\section{The use of palmitoylethanolamide (PEA) in patients suffering from neuropathic pain due to CIAP}

In the absence of clinical guidelines on how to treat neuropathic pain in CIAP patients, many physicians prescribe the classical analgesics such as amitriptyline, pregabalin, and gabapentin. In our clinic, many of the CIAP patients we see who are suffering from neuropathic pain have already been treated with sufficient doses of these drugs. Either patients complain about the intolerable side effects, or the analgesia is not sufficient.

Since 2010, we have explored the therapeutic profile of PEA, an endogenous fatty acid amide, a novel agent in the treatment of chronic neuropathic pain. ${ }^{13,14}$ PEA is available as a food supplement in capsules containing $400 \mathrm{mg}$ micronized PEA (PeaPure, JP Russell Science Ltd., Nicosia, Cyprus), and as food for medical purposes in Italy and Spain, in tablets of 200, 300, 400, and 600 mg (Pelvilen, Normast; Epitech Srl., Milano, Italy). PEA has shown efficacy in many different preclinical animal models for chronic and neuropathic pain. Since 1970 , its safety and efficacy has been explored in a variety of clinical trials in a number of inflammatory and chronic pain states. Since the first paper on PEA was indexed in PubMed in 1968, more than 350 entries have appeared under the keyword "palmitoylethanolamide." The compound was discovered in 1957 by Kuehl et al ${ }^{15}$ and used many decades ago in some countries, but due to a lack of insight into its mechanism of action, interest waned. Since the 1990s, interest has surged again due to the discovery of its mechanism of effect by the Nobel laureate Professor Rita Levi-Montalcini. ${ }^{16,17}$
PEA is a naturally occurring fatty acid amide, belonging to the class of the nuclear factor peroxisome proliferatoractivated receptor (PPAR)-alpha agonists. In addition to its affinity for PPAR-alpha, it has high affinity for the GPR55 receptor. ${ }^{18-20}$ PEA and related acyl-ethanolamides are also involved in additional mechanisms of action, such as influencing TRPV1, ion channels, and inhibiting nitric oxides. ${ }^{21}$ Previously, PEA was characterized as an endocannabinoid, but PEA has no affinity for cannabinoid receptors 1 or 2 . Amide lipids such as PEA are widely present in nature, in a variety of plant, invertebrate, and mammalian tissues. PEA is also present in many food products, such as peanuts, egg yolk, and soy beans and has been explored since the 1970s in a great number of clinical trials, as summarized by Keppel Hesselink. ${ }^{13,14}$

Clinical research on PEA started in the 1960s and 1970s, especially in the Czech Republic. PEA at that time was commercially available as far as we know as a non-micronized matrix tablet formulation under the brand name Impulsin, and was indicated for prevention of flu and respiratory diseases as well as for immune system enhancement. Many years have passed since, with PEA being subsequently explored in a variety of pain states: diabetic neuropathy, carpal tunnel syndrome, dental and temporomandibular joint pain, and arthritic, postherpetic, and chemotherapy-induced neuropathic pain. The results from a number of human clinical pain trials have been discussed previously. ${ }^{13}$ Overall, more than 5,000 patients have been successfully treated with PEA in the context of all clinical trials, and no adverse effects reported in any of these trials..$^{13,22}$

\section{Experience with PEA in neuropathic pain in CIAP: a case collection}

We selected a number of pain treatment-resistant, laboratory, and electrophysiological confirmed cases of CIAP patients who visited our clinic, and started adding PEA to the analgesic treatment regime, or substituted PEA for the analgesic drugs on request of the patients, mostly related to intolerable side effects, such as drowsiness and concentration difficulties.

This paper presents a case collection of seven patients suffering from CIAP, selected based on the availability of the diagnosis supported by classical electromyographic (EMG) findings of axonal neuropathy and per exclusionem of other cases via laboratory evaluation (eg, vitamin B12 deficiency, vitamin B6 intoxication, inflammatory neuropathy, etc), according to the Dutch Guideline for polyneuropathy evaluation. ${ }^{23}$ All patients showed a clear beneficial effect 
of PEA. Six patients were treatment-refractory patients, and we added a special patient to this series. One of the patients, patient 6 , was analgesic naïve, and wished to start treatment with pharmacotherapy devoid of central nervous system side effects, which is why we started treatment with PEA.

\section{Patient I}

An 81-year-old female patient (born in 1932) started to suffer from pain in both feet in 2003; pain was progressive in nature. We saw this patient first in 2013 ; her pain score was 8 over 10 on the numeric rating scale (NRS) when she visited our clinic. The pain was located in both legs from the knees downwards, and the patient experienced subjective feelings as if walking on a pillow. Clinical examination showed absence of Achilles tendon reflex and absence of vibration sense in the feet up to the knee. Previous neuropathic pain treatments did not result in any pain reduction, neither amitriptyline $50 \mathrm{mg}$ nor duloxetine $30 \mathrm{mg}$. The diagnosis axonal polyneuropathy was confirmed by electrophysiological examination in 2008 . Symptoms grew worse, and increasingly she complained about strange feelings in both feet, as if walking on a ball, dead feelings of toes, and difficulty walking due to pain. She did not wish to be treated with pregabalin or opioids. We started treating with palmitoylethanolamide (PeaPure), $400 \mathrm{mg}$ three times daily. Within 7 days, pain scores were reduced by $50 \%$, and after 2 weeks the patient reported absence of pain. No side effects were reported.

\section{Patient 2}

A 55-year-old Caucasian male (born in 1957) started to suffer from pain in both feet around 20 years ago, which was initially treated by a podologist. Some years later, numbness started, especially noticeable during bursts of activity, and playing tennis became impaired. In 2008, neurologists diagnosed the patient as suffering from CIAP, based on an electrophysiological examination. Analgesic treatment with gabapentin was suggested, but the patient declined treatment due to the side effect profile. In a few years, pain grew worse up to 8 over 10 on the 11-point NRS (0-10), and treatment was started with pregabalin, $75 \mathrm{mg}$ bid. This gave a reduction of pain of around $40 \%$, with the NRS score decreasing from 8 to 5 . However, the patient was unsatisfied due to side effects, especially drowsiness, difficulties concentrating, and an inability to drive a car. However, after stopping pregabalin, pain grew again in intensity to a score of 8 on the NRS. He then visited our clinic. We started treatment with PEA, $400 \mathrm{mg}$ three times daily. After 2-3 weeks on PEA, pain scores were down to 4 on the NRS. The patient's quality of life improved, as did his sleep pattern, and he remained stable until we discharged him several months later. No side effects were reported.

\section{Patient 3}

A 66-year-old Caucasian male (born in 1947) suffered from chronic idiopathic axonal polyneuropathy, and showed a mean pain score of 8 on the NRS. The diagnosis was supported by EMG analysis in 2011, showing signs of a severe axonal neuropathy, without any sign of demyelinization. The complaints started in 2000, with burning and tingling in both feet. Neuropathic pain started after walking 10 miles and more. Being an enthusiastic walker, he started to feel very annoyed by the symptoms. He refused to take analgesics such as amitriptyline and pregabalin because of much-feared side effects. We started treatment with PEA according to our protocol, and month-by-month his pain decreased. After 2-3 months, pain was reduced to NRS $1-2$, and the patient was able to start walking long distances again. Side effects were not reported.

\section{Patient 4}

A 73-year-old Caucasian female (born in 1941) suffering from burning pains since 2003 was diagnosed with sensory axonal neuropathy as confirmed by EMG in 2003. Pain scores when we first saw her in 2011 were 8 out of 10 on the NRS. TENS (transcutaneous electrical nerve stimulation) and amitriptyline $30 \mathrm{mg}$ before sleep did not result in pain reduction, tramadol $100 \mathrm{mg}$ bid did help to bring down peak pain effects. Treatment was started using $1200 \mathrm{mg}$ PEA per day, and this reduced the burning pain from NRS score 8 to 6 . The patient stopped tramadol, and this resulted in a relapse of pain. Tramadol was reinitiated, and PEA dose was increased to $2000 \mathrm{mg}$ daily. Pain intensity decreased, and within 14 days, all burning sensations vanished; the patient spoke of "a miracle." The situation remained stable for months, and side effects were not reported.

\section{Patient 5}

A 42-year-old Caucasian female (born in 1969) visited our clinic in 2011, mainly because of severe burning pains and paresthesia due to an axonal neuropathy, diagnosed via EMG in 2011 and 2012. She had also been suffering from numbness in feet and fingers for many years. Although treated with $1200 \mathrm{mg}$ gabapentin daily, her pain score on the NRS was 8 out of 10 when we first saw her. We added $1200 \mathrm{mg}$ PEA daily, and within some weeks, pain was reduced to an NRS score of 3. She started physiotherapy and was able to start running again. Side effects were not reported. 


\section{Patient 6}

A 44-year-old Caucasian female (born in 1967) visited our clinic in 2011. Her medical history included two operations for a Morton's neuroma (1997, 2008). EMG findings supported an axonal neuropathy. Her pain score on the NRS was $6-8$, despite $20 \mathrm{mg}$ amitriptyline and $150 \mathrm{mg}$ pregabalin daily. We added $1200 \mathrm{mg}$ PEA daily, and this resulted in a gradual pain reduction over some weeks, until an NRS score of 2-3. She was then able to wean-off from pregabalin and amitriptyline, without pain relapses. The patient has remained stable since, and side effects were not reported.

\section{Patient 7}

A 63-year-old Caucasian female (born in 1948) visited our clinic in 2011. She was diagnosed as suffering from a severe sensory and motor axonal neuropathy, based on EMG findings. Her medical history included an L5 discus prolapse that was operated upon. Apart from sensory complaints, there was a severe burning pain and instability, as well as drop feet and pareses of feet extensors and toe extensors (Medical Research Council grade 4). We started treating with $1200 \mathrm{mg}$ PEA daily, and pain decreased from 7 on the NRS score to a mean score of 3, with peaks up to NRS score of 5. Side effects were not reported.

\section{PEA treatment schedule and PEA formulations}

All the patients described above were responders on $1200 \mathrm{mg}$ PEA per day. In our clinic, we see predominantly polyneuropathic pain patients, most of them suffering from diabetic polyneuropathy or chemotherapy-induced polyneuropathic pain. In general, we adhere in our clinic to a standardized dosing regimen of PEA in cases of polyneuropathic pain, starting with $1200 \mathrm{mg} /$ day (1-2 capsules PEA in its excipientfree micronized formulation [PeaPure] 2-3 times per day).
For instance, in cases of severe pain during the night, we suggest taking two capsules after dinner and one after breakfast, and if most pain is experienced during the day we suggest taking two capsules in the morning after breakfast and one after the evening meal. We keep patients on a step-in dose of $1200 \mathrm{mg} /$ day and in case of good response (30\%-50\% pain reduction on the NRS within 2 weeks), we keep the dose stable. However, if pain reduction is less satisfactory, we titrate upwards to a maximum of $30 \mathrm{mg}$ PEA per kilogram bodyweight. We have used this dose in hundreds of patients, without side effects. Recently, Esposito et $\mathrm{al}^{24}$ reported that doses of $100 \mathrm{mg}$ per kilogram bodyweight in adults and $50 \mathrm{mg}$ per kilogram bodyweight in children were tolerated without safety concerns.

There are currently a number of formulations available, containing PEA in different amounts, micronized and unmicronized, and three formulations contain in addition to PEA various herbal extracts or components. These products are summarized in Table 1, in order of decreasing PEA content.

We use in our clinic the micronized PEA formulation without excipients and without herbal additions, as we prefer a micronized PEA product, free from excipients such as magnesium stearate, povidone, sorbitol, and/or herbal additions, due to the fact that such excipients and additions might decrease the bioavailability of fatty compounds such as PEA, and might lead to gastrointestinal complaints, such as diarrhea and gastric discomfort, and other side effects. ${ }^{25-28}$

In addition to these considerations, the clinical relevance of adding herbal components to PEA formulations is unclear, as there are no comparative studies between PEA products with and without these additions.

Finally, this choice is due to the pragmatic fact that this formulation is commercially available in the Netherlands, while the other micronized PEA products are not.

Table I PEA products summarized, in order of decreasing PEA content

\begin{tabular}{llll}
\hline Product name and dose & Formulation & \% PEA & Contents \\
\hline PeaPure $400 \mathrm{mg}$ & Micronized capsules & $100 \%$ & PEA only \\
Normast $300 \mathrm{mg}$ & Micronized matrix tablet & $68 \%$ & PEA, excipients \\
Normast $600 \mathrm{mg}$ & Micronized matrix tablet & $68 \%$ & PEA, excipients \\
Normast $600 \mathrm{mg}$ & Micronized sachets, sublingual use & $60 \%$ & PEA, excipients (sorbitol $384 \mathrm{mg})$ \\
Pelvilen Forte $400 \mathrm{mg}$ & Coated micronized matrix tablet & $60 \%$ & PEA, excipients, trans-polidatin (40 mg) \\
Pelvilen $200 \mathrm{mg}$ & Coated micronized matrix tablet & $49 \%$ & PEA, excipients, trans-polidatin (20 mg) \\
Glialia $700 \mathrm{mg}$ & Micronized sachets, sublingual use & $47 \%$ & PEA, excipients (sorbitol $483 \mathrm{mg})$, luteolin (70 mg) \\
Achilles $825 \mathrm{mg}$ & Unmicronized matrix tablet & $12 \%$ & PEA, excipients, extracts of Cissus quadrangularis, \\
& & & Zingiber zerumbet, and Siegesbeckia orientalis \\
\hline
\end{tabular}

Abbreviation: PEA, palmitoylethanolamide. 


\section{Conclusion}

In our case series, we saw good to excellent pain reduction with PEA (either alone or combined with other treatments) in seven EMG-confirmed CIAP patients. Combinations with regular analgesics did not lead to drug-drug interactions, and in all seven patients, PEA tolerability was excellent. As patients were treatment-resistant to previous analgesics, we conclude it is highly likely the good clinical response is more than placebo effect alone.

PEA represents a promising addition to our therapeutic armamentarium for neuropathic pain caused by CIAP, with good tolerability and a broad safety margin. It therefore warrants wider use in this patient group, where alternatives are quite limited and often cause cumbersome side effects.

\section{Disclosure}

The author reports no conflicts of interest in this work.

\section{References}

1. Fisher CM. Late-life chronic peripheral neuropathy of obscure nature. Arch Neurol. 1982;39:234-235.

2. McLeod JG, Tuck RR, Pollard JD, Cameron J, Walsh JC. Chronic polyneuropathy of undetermined cause. J Neurol Neurosurg Psychiatry. 1984;47:530-535.

3. Notermans NC, Wokke JHJ, van der GraafY, Franssen H, van Dijk GW, Jennekens FGI. Chronic idiopathic axonal polyneuropathy: a five year follow up. J Neurol Neurosurg Psychiatry. 1994;57:1525-1527.

4. Marchiori PE, Lima A, Hirata MTA, Scaff M. Chronic axonal inflammatory polyneuropathy after (CAIP) after transplantation. J Neurol Sci. 1997;150 Suppl 1:S242.

5. Vrancken AF, van Schaik IN, Hughes RA, Notermans NC. Drug therapy for chronic idiopathic axonal polyneuropathy. Cochrane Database Syst Rev. 2004;(2):CD003456.

6. Singer MA, Vernino SA, Wolfe GI. Idiopathic neuropathy: new paradigms, new promise. J Peripher Nerv Syst. 2012;17 Suppl 2: 43-49.

7. Visser NA, Vrancken AF, van der Schouw YT, van den Berg LH, Notermans NC. Chronic idiopathic axonal polyneuropathy is associated with the metabolic syndrome. Diabetes Care. 2013;36:817-822.

8. Tierney EF, Thurman DJ, Beckles GL, Cadwell BL. Association of statin use with peripheral neuropathy in the US population 40 years of age or older. J Diabetes. 2013;5:207-215.

9. Murinson BB, Haughey NJ, Maragakis NJ. Selected statins produce rapid spinal motor neuron loss in vitro. BMC Musculoskelet Disord. 2012;13:100

10. Vural K, Tuğlu MI. Neurotoxic effect of statins on mouse neuroblastoma NB2a cell line. Eur Rev Med Pharmacol Sci. 2011;9:985-991.
11. Erdmann PG, van Genderen FR, Teunissen LL, et al. Pain in patients with chronic idiopathic axonal polyneuropathy. Eur Neurol. 2010;64:58-64.

12. de Schryver EL, van Schelven LJ, Notermans NC, de Valk HW, Oey PL. Small-fibre neuropathy can be detected in patients with chronic idiopathic axonal polyneuropathy. Eur J Neurol. 2011;18:1003-1005.

13. Keppel Hesselink JM. New targets in pain, non-neuronal cells, and the role of palmitoylethanolamide. Open Pain J. 2012;5:12-23.

14. Keppel Hesselink JM, Hekker TA. Therapeutic utility of palmitoylethanolamide in the treatment of neuropathic pain associated with various pathological conditions: a case series. J Pain Res. 2012;5:437-442.

15. Kuehl FA, Jacob TA, Ganley OH, Ormond RE, Meisinger MAP. The identification of N-2-hydroxyethyl-palmitamide as a natural occurring antiinflammatory agent. J Am Chem Soc. 1957;79:5577-5578.

16. Aloe L, Leon A, Levi-Montalcini R. A proposed autacoid mechanism controlling mastocyte behaviour. Agents Actions. 1993;39: C145-C147.

17. Keppel Hesselink JM. Professor Rita Levi-Montalcini on nerve growth factor, mast cells and palmitoylethanolamide, an endogenous antiinflammatory and analgesic compound. J Pain Relief. 2013;2(1):1000114.

18. Khasabova IA, Xiong Y, Coicou LG, Piomelli D, Seybold V. Peroxisome proliferator-activated receptor alpha mediates acute effects of palmitoylethanolamide on sensory neurons. J Neurosci. 2012;32: 12735-12743.

19. LoVerme J, La Rana G, Russo R, Calignano A, Piomelli D. The search for the palmitoylethanolamide receptor. Life Sci. 2005;77:1685-1698.

20. Godlewski G, Offertaler L, Wagner JA, Kunos G. Receptors for acylethanolamides-GPR55 and GPR119. Prostaglandins Other Lipid Mediat. 2009;89(3-4):105-111.

21. Costa B, Comelli F, Bettoni I, Colleoni M, Giagnoni G. The endogenous fatty acid amide, palmitoylethanolamide, has anti-allodynic and antihyperalgesic effects in a murine model of neuropathic pain: involvement of CB(1), TRPV1 and PPARgamma receptors and neurotrophic factors. Pain. 2008;139:541-550.

22. Keppel Hesselink JM, de Boer T, Witkamp R. Palmitoylethanolamide: a natural body-own anti-inflammatory agent, effective and safe against influenza and common cold. Int J Inflammation. In press 2013.

23. Doorn PA. Richtlijn 'Polyneuropathie' [Guideline on polyneuropathy]. Ned Tijdschr Geneeskd. 2007;151:1566-1573. Dutch.

24. Esposito E, Cuzzocrea S. Palmitoylethanolamide is a new possible pharmacological treatment for the inflammation associated with trauma. Mini Rev Med Chem. 2013;13(2):237-255.

25. Gould M, Sellin JH. Diabetic diarrhea. Curr Gastroenterol Rep. 2009;11:354-359.

26. Vernia P, Frandina C, Bilotta T, Ricciardi MR, Villotti G, Fallucca F. Sorbitol malabsorption and nonspecific abdominal symptoms in type II diabetes. Metabolism. 1995;44:796-799.

27. Uchimoto T, Iwao Y, Takahashi K, et al. A comparative study of glycerin fatty acid ester and magnesium stearate on the dissolution of acetaminophen tablets using the analysis of available surface area. Eur J Pharm Biopharm. 2011;78:492-498.

28. Levy G, Gumtow RH. Effect of certain tablet formulation factors on dissolution rate of the active ingredient. III. Tablet lubricants. J Pharm Sci. 1963;52:1139-1144.
International Medical Case Reports Journal

\section{Publish your work in this journal}

The International Medical Case Reports Journal is an international, peer-reviewed open-access journal publishing original case reports from all medical specialties. Previously unpublished medical posters are also accepted relating to any area of clinical or preclinical science. Submissions should not normally exceed 2,000 words or

\section{Dovepress}

4 published pages including figures, diagrams and references. The manuscript management system is completely online and includes a very quick and fair peer-review system, which is all easy to use. Visit http://www.dovepress.com/testimonials.php to read real quotes from published authors. 